\title{
Persistence in Cluster-Cluster Aggregation
}

\author{
E. K. O. Hellén and M. J. Alava \\ Helsinki University of Technology, Laboratory of Physics, P.O.Box 1100, FIN-02015 HUT, Finland
}

(November 3, 2018)

\begin{abstract}
Persistence is considered in diffusion-limited cluster-cluster aggregation, in one dimension and when the diffusion coefficient of a cluster depends on its size $s$ as $D(s) \sim s^{\gamma}$. The empty and filled site persistences are defined as the probabilities, that a site has been either empty or covered by a cluster all the time whereas the cluster persistence gives the probability of a cluster to remain intact. The filled site one is nonuniversal. The empty site and cluster persistences are found to be universal, as supported by analytical arguments and simulations. The empty site case decays algebraically with the exponent $\theta_{E}=2 /(2-\gamma)$. The cluster persistence is related to the small $s$ behavior of the cluster size distribution and behaves also algebraically for $0 \leq \gamma<2$ while for $\gamma<0$ the behavior is stretched exponential. In the scaling limit $t \rightarrow \infty$ and $K(t) \rightarrow \infty$ with $t / K(t)$ fixed the distribution of intervals of size $k$ between persistent regions scales as $n(k ; t)=K^{-2} f(k / K)$, where $K(t) \sim t^{\theta}$ is the average interval size and $f(y)=e^{-y}$. For finite $t$ the scaling is poor for $k \ll t^{z}$, due to the insufficient separation of the two length scales: the distances between clusters, $t^{z}$, and that between persistent regions, $t^{\theta}$. For the size distribution of persistent regions the time and size dependences separate, the latter being independent of the diffusion exponent $\gamma$ but depending on the initial cluster size distribution.
\end{abstract}

PACS numbers: 05.40.-a, 82.20.Mj, 05.50.+q, 05.70.Ln, 02.50.Ey

\section{INTRODUCTION}

Persistence in dynamical systems has attained considerable interest recently [1]. It was originally introduced for a simple diffusion process [2,3] but since then it has been studied, for example, in spin systems [4] reaction-diffusion systems [13 19], voter model [20,21] and for interfaces 22 25. It has also been measured experimentally 26 30, and a few exact results exist 31.34.

The persistence is usually defined as the probability $P(t)$ that at a fixed point in space a fluctuating nonequilibrium field $\phi(x ; t)$ does not change sign upto time $t$, i.e., the probability that $\operatorname{sgn}[\phi(x ; t)-\langle\phi(x ; t)\rangle]$ remains unchanged. In many systems the probability decays algebraically, $P(t) \sim t^{-\theta}$, with a nontrivial persistence exponent $\theta$. The significance of the phenomenon stems from the fact that the exponent is not, in general, related to the usual static or dynamic exponents. This this in turn implies that not necessarily all of the properties of the system are characterized by a single length scale.

In this article we study various definitions of persistence in an aggregation process, in the disguise of the one-dimensional diffusion-limited cluster-cluster aggregation model (DLCA) 35, with each cluster diffusing with a size dependent diffusion coefficient, $D(s) \sim s^{\gamma}$ with $\gamma<2$. This model is in many respects suitable for studying persistence as it possesses a rich dynamical behavior but at the same time it is simple enough to often allow for an analytical treatment [36]. Moreover in DLCA one meets immediately the possibility of defining several persistent quantities, each of which describes a different aspect of the aggregation process.

The persistence probabilities considered in this work are i) the probability of a cluster to remain unaggregated (cluster persistence), ii) the probability that a site has been empty (empty site persistence) and iii) filled (filled site persistence) upto time $t$. Notice that all these are local quantities. It is possible, in analogy to e.g. the contact process [37], to work starting from definitions that involve a global quantity like the average cluster size. Thus one could compare the ensemble average of the cluster size to that in any particular sample and investigate the sign changes of their difference. Likewise one could attempt the same, a comparison to the average cluster size, for all individual clusters 38. These would be, in practice, much harder to study numerically than i) to iii) above which also have the pleasant aspect of being, possibly, experimentally relevant. The comparisons between the three quantities points out the underlying mechanisms, which make some persistent quantities to be universal while the others are not.

Recently Manoj and Ray claimed that the dynamic exponent associated with the growth of the intervals between two consecutive persistent sites in a diffusionannihilation model $A+A \rightarrow \emptyset$ shows nonuniversal behavior, i.e., the exponent depends on the initial concentration of particles [14. This started a discussion [15, 16], concerning for example whether it matters if only the empty sites are taken to be persistent at $t=0$ instead of the entire lattice. The problem of universality remained unsolved. Later on, the same authors argued that the reason might be the competition between the two length scales, the diffusive scale $\mathcal{L}_{D}$ and the persistence length scale $\mathcal{L}_{p}$ [17,18. Very recently, Bray and O'Donoghue studied a similar problem in the $q$-state Potts model [4]. They found no evidence on the concentration dependence and showed, that the characteristic length scale 
$\mathcal{L}_{c}$ controlling the distribution of the intervals between persistent sites, is given by the maximum of the two: $\mathcal{L}_{c}=\max \left\{\mathcal{L}_{D}, \mathcal{L}_{p}\right\}$. Furthermore they argued that the poor scaling for small values of $q$ is due to the insufficient separation of these two length scales. These issues are easier to resolve in the DLCA where the length scale separation is more pronounced.

First we discuss the three persistence definitions with size independent diffusion coefficients $(\gamma=0)$ in order to clarify the universality of these quantities. The filled site persistence turns out to be nonuniversal whereas the two others decay similarly independent of the concentration and initial conditions. This difference between empty and filled site persistence is in clear contrast to all the previous persistence studies of, for example, coarsening in the Ising model [7], where the symmetry between up and down spins is not broken.

Thereafter we concentrate on the two universal persistent quantities and consider the influence of size dependent diffusion $(\gamma \neq 0)$. We discover that it is possible to have situations, in which the other persistence decays faster than any power-law in time whereas the other shows algebraic behavior. At the end we study the distributions of the persistent regions and the intervals between these. The region size distributions depend on the initial cluster size distribution. The interval size distributions are universal, simple exponentials, and their scaling nicely demonstrates the effect of the presence of the two length scales, $\mathcal{L}_{p}$ and $\mathcal{L}_{D}$.

This paper starts by introducing the model and describing the quantities of interest in section II. The known results for the persistence probabilities are presented in the beginning of section III. In the rest of that section each persistence probability is analyzed separately. Section IV discusses the scaling of the region and interval size distributions. The dependence of the persistent quantities on concentration and initial conditions are studied by simulations in the beginning of section $\mathrm{V}$. The end of that section shows the numerical results for the region and interval size distributions. Section VI concludes the paper.

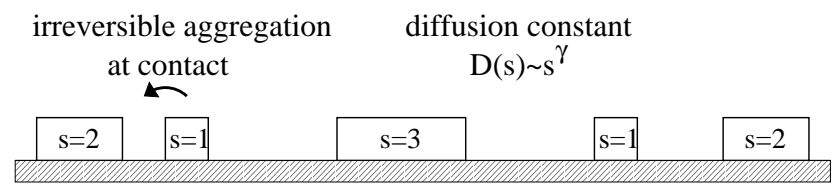

FIG. 1. Illustration of the one-dimensional DLCA model.

\section{MODEL AND QUANTITIES OF INTEREST}

The diffusion-limited cluster-cluster aggregation model is here considered on a one-dimensional lattice of $L$ sites with periodic boundary conditions. Initially the lattice is filled upto a concentration $\phi$ such that occupied lattice sites correspond to particles and sites connected via nearest neighbor occupancy belong to the same cluster. Each cluster performs a random walk such that the diffusion coefficient of a cluster of size $s$ is given by $D(s)=D_{1} s^{\gamma}$. The positive constant $D_{1}$ sets the time scale and it is irrelevant regarding the dynamic scaling properties of the system which are controlled by the diffusion exponent $\gamma \quad 39$ 42. Here we concentrate on $\gamma<2$ for which the growth of the average cluster size is algebraic. For $\gamma>2$ the system will gel, i.e., there will be an infinite cluster at a finite time. When two clusters collide, they aggregate irreversibly together. If the sizes of the colliding clusters were $s$ and $s^{\prime}$, the aggregate is of size $s+s^{\prime}$ and its diffusion constant $D\left(s+s^{\prime}\right)$. Figure 1 visualizes the model.

The one-dimensional DLCA is closely related to other models when the diffusion coefficient of a cluster is independent of its size, i.e., $\gamma=0$. For example, consider the zero temperature one-dimensional $q$-state Potts model with Glauber dynamics. The different spin species are separated by domain walls, each of which performs a simple random walk. By representing each such a walker by a particle $A$, the two particles colliding react according to

$$
A+A \rightarrow\left\{\begin{array}{lll}
A & \text { with probability } & (q-2) /(q-1) \\
\emptyset & \text { with probability } & 1 /(q-1)
\end{array}\right.
$$

In the limit $q \rightarrow \infty$ this reduces to the reaction-diffusion model $A+A \rightarrow A$, which can be solved exactly [43]. The same is true for particles with a finite mass $A_{i}+A_{j} \rightarrow$ $A_{i+j}$ [36]. The only difference between this and the DLCA model is the finite extent of clusters in the DLCA. It has no relevance on the cluster size distribution but the difference may be essential for persistence. This is to be expected since i) the $q$-state Potts model can be mapped to the Ising model $(q=2)$ with an initial fraction $1 / q$ of up spins [8] and ii) the persistence exponent of the Ising model is known to depend on the concentration [6.6]. Note, that in the Potts model the usual definition for persistence characterizes the fraction of spins, which have remained in their original state.

There are many ways to define persistence in clustercluster aggregation. Perhaps the most closest to the definition used in the context of Ising systems would be the one which relates to the question of site persistence: what is the probability, that a site has been either covered or uncovered by a cluster for all times $[0, t]$ ? However, in DLCA there are also other natural candidates. 


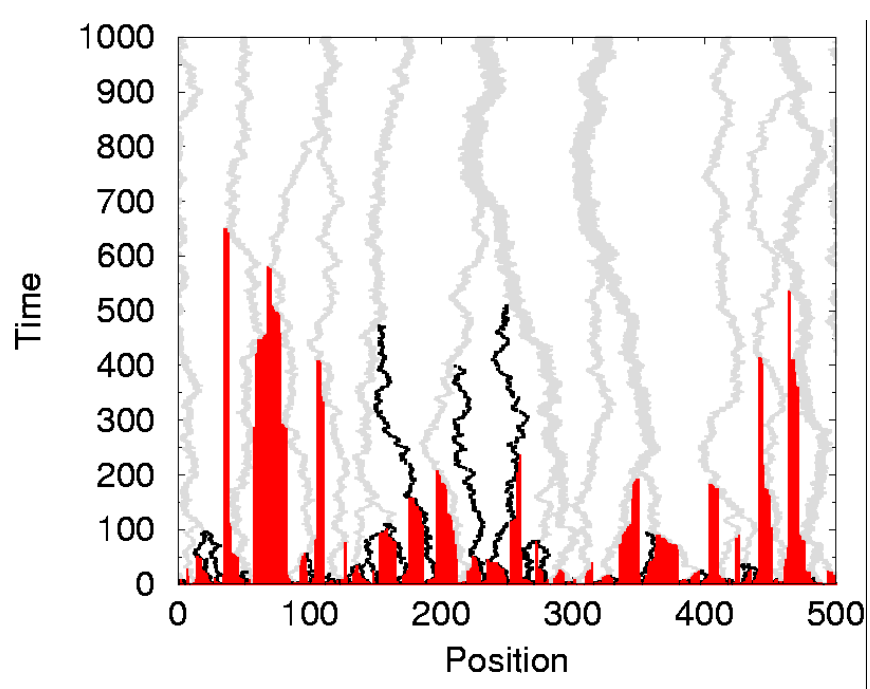

FIG. 2. A space-time plot of the dynamics in DLCA for $\gamma=0$. The clusters are denoted by light gray, the persistent clusters by black and the persistent empty regions by dark grey.

The persistence probabilities studied in this work are the following:

- Cluster persistence: the probability that a cluster has not aggregated, $P_{C}(t) \sim t^{-\theta_{C}}$.

- Filled site persistence: the probability that a site originally covered by a cluster has been covered by it all the time, $P_{F}(t) \sim t^{-\theta_{F}}$.

- Empty site persistence: the probability that an originally empty site has never been occupied by a cluster, $P_{E}(t) \sim t^{-\theta_{E}}$.

When the persistence probabilities decay algebraically, the associated decay exponents $\theta_{C}, \theta_{F}$, and $\theta_{E}$ become persistence exponents. Figure 2 shows an example of the dynamics together with the persistent clusters and the persistent empty sites for a small system.

In the following we will concentrate only on the three definitions given above. However, some other persistences can be directly obtained from the results given. For example, the aforementioned site persistence probability is defined as the probability that a site has remained in the same state, i.e. either filled or empty, all the time and is given by $P_{S}(t)=P_{F}(t)+P_{E}(t)$. The survival of domains follows from the studies of the persistent region size distribution (defined below). This is a special case of the so called adaptive persistence [44].

Furthermore one could consider the particle persistence, which is the probability that a site has remained either empty or covered by the same particle all the time. Recall that a cluster of size $s$ consist of $s$ particles. The probability that a cluster has not moved before time $t$ decays exponentially making particle persistence a rather trivial quantity.
The cluster persistence differs drastically from the other persistence definitions. It is not a quantity defined per a fixed site on the lattice as the others are but is a property associated with each cluster. Due to that also its behavior is rather different than that of the other persistences considered. However, it is a rather similar, though not exactly the same, quantity as the domain persistence in the Ising model [6.7. For pointlike clusters it leads to a study of survival of coalescing random walks. A similar study has very recently been carried out for non-interacting walks and for the reaction-diffusion system $A+B \rightarrow \emptyset$ in [19] and for the annihilation-coalescence reaction-diffusion system corresponding to the Potts model [Eq.(1)] in [12].

In addition to the persistence probabilities we will also study distributions associated with the persistent sites and the distances between them. The terminology and notation used are as follows. The word region is reserved for a bunch of consecutive persistent sites. The distances between regions, i.e., between two consecutive persistent sites, is called an interval. The word cluster has the obvious meaning. The number of clusters of size $s$ per lattice site at time $t$ is $n_{s}(t)$ with the normalization $\sum_{s} s n_{s}=1$. Region size is denoted by $l$ and the number of regions of size $l$ (per site) by $p_{X}(l ; t)$. The subscript is like for the persistence probabilities and it refers to the persistence definition used: $X \in\{C, F, E\}$. When using the continuum description we use the symbol $r$ instead of $l$. The letter $k$ labels the interval sizes and the corresponding distribution function is $n_{X}(k ; t)$. The number densities of persistent sites (the persistence probability) and nonpersistent ones are denoted by capital letters $P_{X}(t)$ and $N_{X}(t)$, respectively.

As an example, consider intervals between persistent empty sites and their distribution function $n_{E}(k ; t)$. The corresponding distribution of persistent regions is $p_{E}(l ; t)$. The number densities are obtained by summing

$$
\begin{aligned}
& N_{E}(t)=\sum_{k=1}^{\infty} k n_{E}(k ; t) \\
& P_{E}(t)=\sum_{l=1}^{\infty} l p_{E}(l ; t) .
\end{aligned}
$$

Obviously these two are related by the equation $P_{E}(t)=$ $1-N_{E}(t)$. Similar formulas apply to other persistence definitions, too, except in the cluster persistence case equation (3) is replaced by

$$
P_{C}(t)=\sum_{l=1}^{\infty} p_{C}(l ; t)
$$

and naturally $P_{C}(t) \neq 1-N_{C}(t)$.

\section{PERSISTENCE PROBABILITIES}

In this section we first list the known exact results for $\gamma=0$. To discuss the cluster persistence we use the 
known results of the dynamic scaling of the cluster size distribution. We also briefly comment on how the cluster persistence exponent is related to the polydispersity exponent, which characterizes the behavior of the scaling function of the cluster size distribution. Then we apply mean-field type continuum methods to analyze the empty site persistences for $\gamma \neq 0$ where no exact results are available. The filled site persistence is analyzed in a similar mean-field fashion in the limits $\phi \rightarrow 0$ and $\phi \rightarrow 1$. This section ends to a discussion on the concentration dependence of the filled site persistence.

\section{A. Known Exact Results}

For the cluster persistence the finite extent of the clusters does not matter and we may replace them by pointlike random walkers. Then we can utilize the results obtained for the the reaction-diffusion model $A_{i}+A_{j} \rightarrow$ $A_{i+j}$. For spatially uncorrelated initial mass distribution $n_{s}(0)=\delta_{s, 1}$ the cluster size distribution is given by $n_{s}(t)=2 s e^{-\xi} I_{s}(\xi) / \xi$, where $\xi=4 D_{1} t$ and $I_{s}(\xi)$ is the modified Bessel function 36$]$. As $n_{1}(t) \sim t^{-3 / 2}$ the cluster persistence exponent $\theta_{C}(\gamma=0)=3 / 2$ in this case. This result is also valid independent of the initial condition as long as the cluster size distribution decays rapidly for large sizes.

The result $\theta_{C}(0)=3 / 2$ can also be obtained without solving the cluster size distribution for general initial size distribution of clusters. Consider a cluster initially at the origin. We want to find the probability that it has not collided with neither of its neighboring clusters before time $t$. As the diffusion coefficient of a cluster does not depend on its size, we do not have to care if the neighboring clusters have collided with other clusters or not. Therefore the problem reduces to the survival probability of three annihilating random walkers. This can solved in several ways [45,5] with the result $\theta_{C}(\gamma=0)=3 / 2$. Unfortunately, these methods rely on the indistinguishability of clusters and can not simply be generalized to the general case of size dependent diffusion coefficients. In fact, even the survival of three annihilating random walkers with constant but non-equal diffusion coefficients is highly nontrivial [46]. In DLCA it is the specific interaction between clusters, the aggregation at contact, that makes the problem simple. The case where a single cluster is hunted by independent clusters is in fact more complicated than our situation with aggregating clusters (see e.g. 47]).

Let us now turn to site persistence when $\gamma=0$. For the empty site persistence the finite extent of the clusters does not matter since a persistent interval is deleted by the edges of neighboring clusters. For finite size clusters the empty intervals are shorter but the length distribution remains the same. The movement of the edges is uncorrelated and unaffected by the collisions with other clusters. These facts make the problem exactly solvable

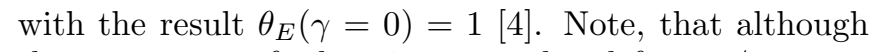
the movement of edges is uncorrelated for $\gamma \neq 0$, too, one can no more ignore the collisions of the neighboring clusters with other clusters. This makes the exact calculation complicated if not intractable. The same applies to the filled site persistence even for $\gamma=0$.

\section{B. Cluster persistence}

Before considering cluster persistence we briefly recapitulate the main results concerning the dynamic scaling in the DLCA. Let the number of clusters of size $s$ per lattice site at time $t$ be $n_{s}(t)$. The cluster size distribution scales as

$$
n_{s}(t)=S(t)^{-2} f(s / S(t)),
$$

where $S(t)$ is the average cluster size and $f(x)$ the scaling function [35]. The diffusive length scale, $\mathcal{L}_{D}$, is proportional to the average cluster size which grows as $S(t) \sim t^{z}$, where $z$ is the dynamic exponent. For a given $\gamma$ both $z$ and the scaling function are universal. In one dimension the dynamic exponent is given by $z=1 /(2-\gamma)$ for $\gamma<2$ and the system gels in a finite time for $\gamma>2$ [40,41]. For small argument values $\left(x \rightarrow 0^{+}\right)$the scaling function $f(x)$ decays as $x^{-\tau}$ for $0 \leq \gamma<2$ and as $\exp \left(-x^{-|\mu|}\right)$ for $\gamma<0$ [39,42]. Though universal, both the exponents $\tau$ and $\mu$ depend nontrivially on $\gamma$. From the small $x$ behavior of the scaling function it follows that $n_{s}(t) \sim s^{-\tau} t^{-w}$ for $1 \ll s \ll S(t)$, where $w=(2-\tau) z$.

The scaling theory further states that for the DLCA all the cluster densities decay in a similar manner at large times: $n_{s}(t) / n_{1}(t) \rightarrow b_{s}$ as $t \rightarrow \infty$ [48. Here $b_{s}$ is a time independent constant. This result together with $n_{s}(t) \sim t^{-w}$ can be directly applied to cluster persistence with a monodisperse initial condition $n_{s}(0)=\delta_{s, s_{0}}$. For simplicity, let all the clusters be monomers, i.e., of unit size, at time $t=0$. Then the cluster persistence probability is equal to $n_{1}(t) \sim t^{-w}$ giving

$$
\theta_{C}=(2-\tau) z
$$

The exact exponents $\tau=-1, z=1 / 2$ [36, and $w=$ $3 / 2$ [45,5] for $\gamma=0$ satisfy this relation. Simulations show that similarly than $z$ and $\tau$ also the persistence exponent is independent of initial conditions and concentration (see Sec. VB).

The result $\theta_{C}=(2-\tau) z$ deserves a few comments. First, since the cluster persistence is a cluster property, it is natural that the persistence exponent is related to the cluster size distribution. Since a persistent cluster has not collided with others, it will definitely be smaller than the average cluster size at late times. Thus the persistent clusters are the ones represented by the small $s$-tail of the cluster size distribution, which is described by the polydispersity exponent $\tau$. On the other hand, if there is any scaling relation between the cluster persistence exponent and the traditional exponents, this should include 
the dynamic exponent. On the basis of this reasoning, the above result is reasonable. However, most of the scaling results quoted above are mean-field of nature and therefore Eq. (6) has to be validated by simulations. The same scaling relation has been found also in the diffusionannihilation model discussed in the Introduction [18].

It follows from the scaling relation that the cluster persistence exponent is as universal as the exponents $z, w$, and $\tau$. However, it does not result in a value for the persistence exponent since $\tau$ is a priori unknown. Calculating it for DLCA and other similar processes has turned out to be a challenging problem since it is given in terms of integrals over the scaling function $f(x)$ itself 39,49 . Also determining the value of $\tau$ reliably in simulations is hard. On the other hand, if one could calculate the persistence exponent and validate the scaling relation one would also obtain $\tau$.

In fact, the persistence exponent can be calculated in a mean-field fashion by replacing the clusters surrounding a persistent cluster by random walkers which diffuse as an average cluster would diffuse. A similar approach works extremely well also for the empty site persistence (see Sec. IIIC). Here it results in studying the survival of three random walkers, whose diffusion coefficients are time-dependent. The meaning of a time-dependent diffusion coefficient in this context is that a random walker will diffuse with a constant diffusion coefficient in another time scale. The detailed analysis and the numerical verification of Eq. (6) will be presented elsewhere [50] and we just give the result here.

It turns out that the cluster persistence probability

$$
P_{C}(t) \sim \begin{cases}\exp \left(-C t^{\beta_{S}}\right) & , \gamma<0 \\ t^{-3 / 2} & , \gamma=0 \\ t^{-2 /(2-\gamma)} & , 0<\gamma<2\end{cases}
$$

with $C>0$ a constant. $\beta_{S}$ fits well to the expression $\beta_{S}=2 / 3(1-2 z)$. Here the completely different behavior of the cluster persistence for $\gamma<0$ and $\gamma \geq 0$ is similar to that of the cluster size distribution which changes character at $\gamma=0$ as noted in the beginning of this Section. The persistence exponent is discontinuous as $\gamma \rightarrow 0^{+}$. Quite unexpectedly, since $\theta_{C}\left(0<\gamma<\frac{2}{3}\right)<\theta_{C}(0)$, it is easier for a cluster to remain persistent if the other clusters will diffuse faster! This somewhat paradoxical result can be understood on the basis that for a random walker, which is hunted by other random walkers, the optimal strategy to survive is to remain stationary (see [51] for a similar situation in the case of noninteracting walkers hunting a single random walker). In the limit $\gamma \rightarrow \infty$ the stretching exponent $\beta_{S} \rightarrow 2 / 3$ is also "discontinuous", since the persistence for a particle bounded by two immobile neighbors decays simply exponentially.

The result (7) together with Eq. (6) implies that also the polydispersity exponent is discontinuous at $\gamma=0$ and, more interestingly, that $\tau(\gamma)=0$ for $0<\gamma<2$. Although this kind of a "superuniversality" is remarkable in its own right, it is more important, that by studying the cluster persistence one learns something about the polydispersity exponent, i.e., the scaling function. This connection may exist in other aggregation models offering a new way to approach the problem of computing the small size tail of the scaling function through a perhaps simpler quantity, the cluster persistence.

\section{Empty site persistence}

Next we give a heuristic argument for obtaining the empty site persistence exponent for arbitrary $\gamma$. Since the clusters on both sides of a persistent empty region are independent, we are led to consider the maximum excursion of a single random walker. The only complication comes from the fact that for $\gamma \neq 0$ the collisions of a cluster with other clusters will change its diffusivity.

Instead of the real process we consider each cluster to have a time-dependent diffusion coefficient $D(t)=$ $D_{0} t^{\gamma z} \sim S(t)^{\gamma}$ with $z=1 /(2-\gamma)$. Then the probability, $P(x ; t)$, to find a cluster at position $x$ at time $t$ obeys a diffusion equation

$$
\partial_{t} P(x ; t)=D_{0} t^{\gamma z} \partial_{x}^{2} P(x ; t) .
$$

A time transformation

$$
T(t)=\frac{D_{0}}{\gamma z+1} t^{\gamma z+1}
$$

reduces equation (8) to an ordinary diffusion equation with the diffusion constant $D=1$. The persistence of the empty space between particles diffusing with a constant $D$ has recently been considered in [4] and we just quote the main results here.

In the long time limit the probability density, $p_{E}(r ; t \mid s)$, that a persistent empty region (originally of size $s$ ) has size $r$ at time $T$ is given by

$$
p_{E}(r ; T \mid s)=\frac{s-r}{\pi T}
$$

and the probability that a cluster survives upto time $t$ is

$$
P_{E}(t \mid s)=\frac{s^{2}}{2 \pi T}=\frac{z}{\pi D_{0}} \frac{s^{2}}{t^{2 z}} \sim t^{-2 z} .
$$

For a general initial length distribution of clusters $n_{s}(0)$ the result will remain the same except $s^{2}$ will be replaced by the average over the initial length distribution $\left\langle s^{2}\right\rangle$.

These considerations show that the persistence exponent $\theta_{E}(\gamma)=2 z=2 /(2-\gamma)$. This agrees with the exact result $\theta_{E}(0)=1$. Furthermore the result is independent of the initial spatial distribution or concentration. All these implications based on the simplified process are confirmed by simulations (see Secs. $\mathrm{VA}-\mathrm{VC}$ ). 


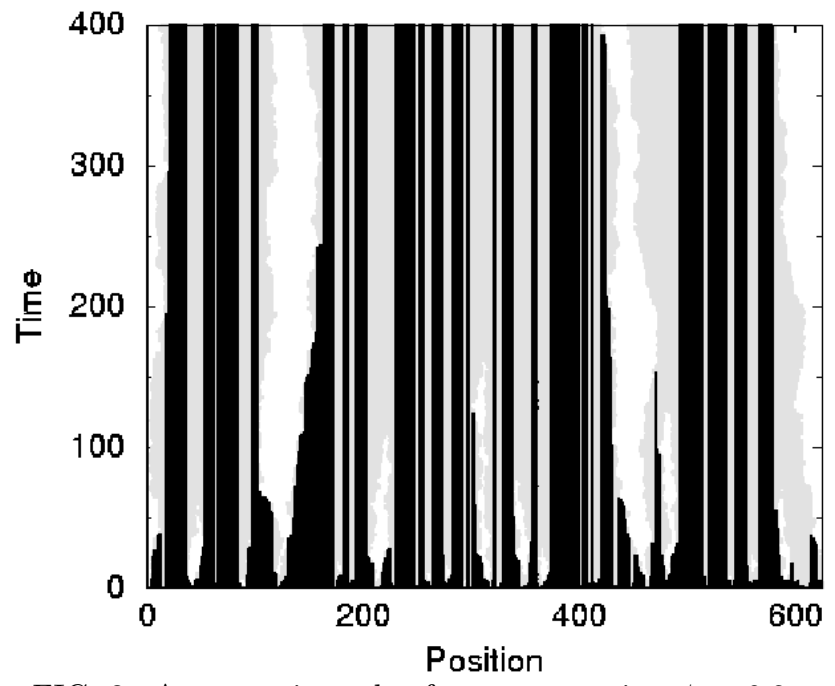

FIG. 3. A space-time plot for concentration $\phi=0.8$ and $\gamma=0$. Cluster are denoted by grey and the persistent empty sites by black.

\section{Filled site persistence}

The behavior of the filled site persistence is different at low and high concentrations. At low concentration a cluster contains typically only one persistent region which is usually destroyed before the cluster aggregates with a neighbor. At high concentrations a large cluster has many persistent regions of various sizes inside it due to the aggregation of clusters (Fig. 3). We now analyze these two limits separately.

In the low concentration limit we consider the persistence problem in continuum, for simplicity. This should be valid for clusters of initial length $l_{0} \gg 1$. For $\phi \rightarrow 0$ the time required for a cluster to move its own length is much smaller than the time required to overcome the distance between clusters. Therefore one could assume that at low concentrations collisions between clusters do not matter. We will see in section $\mathrm{VA}$ that this is true only upto some crossover-time. This time obviously diverges in the limit $\phi \rightarrow 0$. However, to obtain more insight to the empty site persistence we will first ignore the collisions in the following analysis.

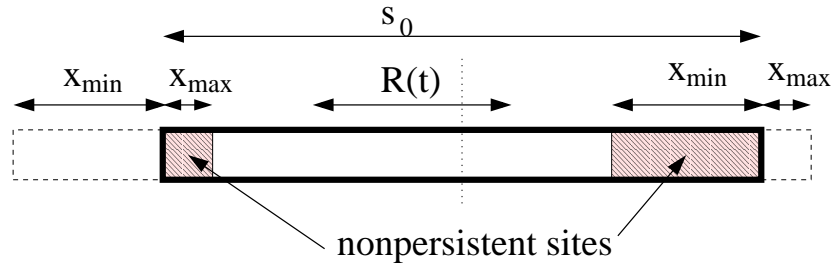

FIG. 4. The length of the nonpersistent filled part is equal to the span $R(t)=x_{\min }(t)+x_{\max }(t)$ of the middle point of a cluster. The length of the persistent region is $s_{0}-R(t)$. The cluster position at $t=0$ is denoted by a thick rectangle, whose middle position is marked by a dotted line. The dashed lines show the maximum excursions of the cluster.
When clusters do not collide, the persistent sites under different clusters are destroyed by independent processes. A single diffusing cluster destroys persistent area at both of its edges. As figure 4 shows, the length of the nonpersistent filled part inside an unaggregated cluster is equal to the span of the middle point. The span, $R(t)$, of a random walk is defined as $R(t)=x_{\min }(t)+x_{\max }(t)$, where $x_{\min }(t)$ and $x_{\max }(t)$ are the maximum displacements of the walk in the negative and positive directions at time $t$, respectively.

The probability distribution for the span of an unlimited random walk is given by [52]

$$
w(R ; t)=\frac{8}{\sqrt{2 \pi D t}} \sum_{j=1}^{\infty}(-1)^{j+1} j^{2} \exp \left(-\frac{j^{2} R^{2}}{2 D t}\right) .
$$

In our case the maximum span is limited by the size of the cluster, $s$, and the probability distribution $p_{F}(r ; t \mid s)$ that an interval of length $r$ of a cluster initially of size $s$ has survived upto time $t$ is

$$
p_{F}(r ; t \mid s)=\frac{8}{\sqrt{2 \pi D t}} \sum_{j=1}^{\infty}(-1)^{j+1} j^{2} \exp \left(-\frac{j^{2}(s-r)^{2}}{2 D t}\right)
$$

for $r \leq s$ and zero otherwise.

We are interested in the asymptotic long time behavior and rather than working directly with the formula in Eq. (13) it is useful to transform it to a more tractable form as follows. Writing the sum in Eq. (13) in the form $\sum_{j=-\infty}^{\infty} G_{j}$ and applying the Poisson sum formula 53.

$$
\sum_{j=-\infty}^{\infty} G_{j}=\sum_{m=-\infty}^{\infty} \int_{-\infty}^{\infty} \mathrm{d} x G(x) e^{2 \pi i m x}
$$

leads to

$$
\begin{aligned}
p_{F}(r ; t \mid s)= & \frac{8 D t}{(s-r)^{3}} \sum_{m=0}^{\infty}\left[\frac{\pi^{2} D t(2 m+1)^{2}}{(s-r)^{2}}-1\right] \\
& \times \exp \left(-\frac{\pi^{2} D t(2 m+1)^{2}}{2(s-r)^{2}}\right) .
\end{aligned}
$$

In Eq. (14) $G(x)$ is an analytic function having the same values at all integers as $G_{j}$.

From Eq. (15) it is easy to show that the probability of finding persistent sites inside the cluster decays exponentially at large times:

$$
\begin{aligned}
p_{F}(t \mid s)= & \int_{0}^{s} \mathrm{~d} r p_{F}(r ; t \mid s) \\
= & \sum_{m=0}^{\infty} \frac{8\left[s^{2}+\pi^{2} D t(2 m+1)\right]}{s^{2} \pi^{2}(2 m+1)^{2}} \\
& \times \exp \left(-\frac{\pi^{2} D t(2 m+1)^{2}}{2 s^{2}}\right) \\
\approx & \frac{8 D t}{s^{2}} \exp \left(-\frac{\pi^{2} D t}{2 s^{2}}\right) .
\end{aligned}
$$


The persistence probability is obtained by integration

$$
p_{F}(t)=\int_{0}^{\infty} \mathrm{d} s p_{F}(t \mid s) n_{s}(0),
$$

so that, in general, the decay will depend on $n_{s}(0)$.

For $n_{s}(0)=\delta\left(s-s_{0}\right)$ it is given by an exponential expression [Eq. (17)] but the change in the decay can be shown using, for example, the initial distribution

$$
n_{s}(0)=\frac{2}{\pi s_{0}} \exp \left(-\frac{s^{2}}{\pi s_{0}^{2}}\right)
$$

whose mean and standard deviation are $\langle s\rangle=s_{0}$ and $\sigma_{s}=\sqrt{\left\langle s^{2}\right\rangle-\langle s\rangle^{2}}=s_{0} \sqrt{(\pi-2) / 2}$, respectively. Using the approximation (17) we get a stretched exponential

$$
p_{F}(t) \approx \sqrt{\frac{128 D t}{\pi^{3} s_{0}^{2}}} e^{-\sqrt{2 \pi D t / s_{0}^{2}}} .
$$

For the Poisson initial distribution, $n_{s}(0)=s_{0}^{-1} e^{-s / s_{0}}$, we can apply the saddle point method to give

$$
p_{F}(t) \approx \sqrt{\frac{128 D t}{3 \pi s_{0}^{2}}} \exp \left(-\frac{3}{2}\left(\frac{\pi^{2} D t}{s_{0}^{2}}\right)^{1 / 3}\right) .
$$

Both of these examples show a stretched exponential decay for the persistence probability, which we believe to be true quite generally. The stretching exponent depends on the initial condition and is therefore nonuniversal. The long time behavior, in the approximation ignoring collisions, is governed by the ratio $D t / s_{0}^{2}$ and we also immediately see that the decay depends on concentration due to the factor $s_{0}$ in the exponential.

The approximative span distribution can be also used to calculate the mean size of persistent regions. First write

$$
\langle r(t \mid s)\rangle=\langle s-R(t \mid s)\rangle=s-\langle R(t \mid s)\rangle
$$

and use the normalized approximate form for the span distribution

$$
\tilde{w}_{N}(R ; t \mid s)= \begin{cases}\delta(R-s)\left[1-\left(1+\xi s^{-2}\right) e^{-\xi / s^{2}}\right] & \\ +2 \xi^{2} R^{-5} e^{-\xi / R^{2}} & ; R \leq s \\ 0 & ; R>s,\end{cases}
$$

where $\xi=\pi^{2} D t / 2$, to calculate $\langle R(t \mid s)\rangle$ from which

$$
\langle r(t \mid s)\rangle \approx s \exp \left\{-\frac{\pi^{2} D t}{2 s^{2}}\right\} .
$$

For the simplest case of a fixed initial size $s_{0}\left[n_{s}(0)=\right.$ $\delta\left(s-s_{0}\right)$ ] the mean length of surviving regions is

$$
\left\langle s_{\text {surv }}\right\rangle=\frac{1}{p_{F}\left(t \mid s_{0}\right)} \int_{0}^{s_{0}} \mathrm{~d} r r p_{F}\left(r ; t \mid s_{0}\right) \approx \frac{2 s_{0}^{3}}{\pi^{2} D t} \sim t^{-1} .
$$

This is just an example and naturally the decay depends on the initial distribution $n_{s}(0)$.

In the high concentration limit, $\phi \rightarrow 1$, we adopt another mean-field type approach: we consider a deterministic model combined with scaling arguments. Let initially the average cluster and empty interval size be $s_{0}$ and size $d_{0}$, respectively. The concentration $\phi=$ $s_{0} /\left(s_{0}+d_{0}\right) \approx 1$ for $s_{0} \gg d_{0}$.

Now consider the doubling times $t_{1}, t_{2}, \ldots, t_{i}, \ldots$ at which the average cluster and interval sizes are $s_{i}=2^{i} s_{0}$ and $d_{i}=2^{i} d_{0}$, respectively. At each step $n$ the doubled cluster is constructed as follows. First, $d_{n-1} / 2$ sites (these does not have to persistent but they may be) from both ends of the cluster are made nonpersistent. Second, the resulting cluster is duplicated. This procedure is repeated. It is quite easy to convince oneself that the probability of finding persistent sites, $p(n)$, at step $n$ decays like $p(n) \sim e^{-\alpha(\phi) n}$ for high enough values of $\phi$. Since $s_{n}=2^{n} s_{0} \sim t^{z}$, it follows that $p(t) \sim t^{-\alpha(\phi) z / \ln 2}$.

According to this simple and crude argument the filled site persistence probability decays algebraically for large enough concentrations. The persistent sites are sweeped by the domain walls (clusters' edges), which get destroyed at aggregation. Since the probability to be touched by a domain wall depends naturally on the density of the walls in the system, the persistence exponent depends on concentration implying nonuniversality. Simulations qualitatively agree with this behavior. We have not made any quantitative comparison since in this paper we concentrate more on the universal properties of the clustercluster aggregation and since being non-universal the decay of site persistence is harder to compute analytically. The simulations furthermore show that the persistence probability decays algebraically for low concentrations, too. The reason for the deviation from the span argument lies in the approximation, which neglects the collisions between clusters. We return to this issue in a more detail in the section $\mathrm{VA}$.

\section{PERSISTENT INTERVAL AND REGION DISTRIBUTIONS}

Having analyzed the various persistence quantities we now turn to the distributions of persistent regions and the intervals between them. First we consider the interval size distributions between consecutive persistent sites and perform a scaling analysis. A natural scaling assumption for these distributions is

$$
n_{X}(k ; t)=K_{X}(t)^{-\alpha} f_{X}\left(k / K_{X}(t)\right),
$$

where $K_{X}(t) \sim t^{\theta_{X}}$ is the average interval size and $X$ denotes any of the choices $C, E$, or $F$. 
Inserting the scaling form (26) to the equation (2) and replacing the sum by an integral (valid in the long time limit) results in

$$
N_{X}(t)=K_{X}(t)^{2-\alpha} \int_{0}^{\infty} \mathrm{d} y y f_{X}(y) .
$$

As $P_{X}(t) \rightarrow 0$ for $t \rightarrow \infty$ it follows from the relation $P_{X}(t)=1-N_{X}(t)$ that $N_{X}(t) \rightarrow 1$ as $t \rightarrow \infty$. The only way to keep the right hand side of Eq. (27) constant is to require $\alpha=2$, as a direct consequence of mass conservation. Note, that the above argument does not require the persistence probability $P_{X}(t)$ to decay algebraically.

Since the persistence exponents are larger than the dynamic exponent, $\theta_{X}>z$, the persistence length scale will be much larger than the diffusive one at large times. This means that the persistent regions are well separated and that they are destroyed by uncorrelated processes since the correlations grow only as $t^{z}$. Each persistent region survives independently of the others and in the scaling limit the scaling functions are therefore simple exponentials

$$
n_{X}(k ; t)=K_{X}(t)^{-2} e^{-k / K_{X}(t)} .
$$

Next let us turn to the size distribution of persistent regions. As the filled site persistence shows nonuniversal behavior, we will here concentrate only on the distributions concerning the empty site and cluster persistence. The former has already been analyzed in section III. For example, equation (10) gives the persistent empty region distribution at large times for a monodisperse initial distribution and for other initial distributions $n_{s}(0)$ it is obtained as

$$
p_{E}(r ; T)=\int_{0}^{\infty} \mathrm{d} s p_{E}(r ; T \mid s) n_{s}(0)=\frac{s_{0}}{\pi T} e^{-r / s_{0}},
$$

where $T$ is given by equation (9) and the last form corresponds to the initial distribution $n_{s}(0)=s_{0}^{-1} e^{-s / s_{0}}$. The dependence on the diffusion exponent enters only through the time scale $T \sim t^{2 /(2-\gamma)}$.

More important is that the spatial and time dependence in $p_{E}(r ; t)$ are decoupled. From this it follows that the average size of the persistent empty regions, $L_{E}(t)$, is a constant at large times. For the monodisperse and Poisson initial conditions we get $L_{E}^{\text {mono }}(t)=$ $P_{E}\left(T \mid s_{0}\right)^{-1} \int_{0}^{s_{0}} \mathrm{~d} r r_{E}\left(r ; T \mid s_{0}\right)=s_{0} / 3$ and $L_{E}^{\mathrm{rand}}(t)=$ $\int_{0}^{\infty} \mathrm{d} r r p_{E}(r ; T) / \int_{0}^{\infty} \mathrm{d} r p_{E}(r ; T)=s_{0}$, respectively. These both are independent of time. The simulations (see Sec. VE) confirm this.

Unfortunately we have not been able to similarly calculate the size distribution of persistent clusters, $p_{C}(l ; t)$. However, for initial cluster size distribution $n_{s}(0)=\delta_{s_{0}, s}$ this distribution remains unaltered. The same is true also for size independent diffusion coefficients $(\gamma=0)$ no matter what the initial distribution is. This does not have to be true when $\gamma \neq 0$. For instance, there may be nontrivial correlations between a persistent cluster and the sizes of clusters surrounding it. The scaling of $p_{C}(l ; t)$ is briefly discussed in section $\mathrm{VE}$.

\section{SIMULATIONS}

The simulations are done on a one-dimensional lattice with periodic boundary conditions. In all the simulations $D_{1}=1$ fixing the time scale. The system sizes range from $5 \cdot 10^{4}$ to $1.5 \cdot 10^{6}$, the data are averaged over $1000-50000$ realizations. The algorithm used is as follows. A cluster is selected randomly and the time is incremented by $N(t)^{-1} D_{\max }^{-1}$, where $N(t)$ is the number of clusters at time $t$ and $D_{\max }$ is the maximum diffusivity of any of the clusters in the system at that time. If $x<D(s) / D_{\max }$, where $x$ is an uniformly distributed random number in the interval $[0,1]$, the cluster is moved with equal probability either to the left or to the right. Otherwise a new cluster is selected and the process is repeated. If a moved cluster collides with another one, the two clusters are aggregated together.

We test the sensitivity of the persistence probabilities against concentration changes (Sec. VA) and two different initial conditions (Sec. VB). The first initial condition used is random, which means that each lattice site is filled with probability $p$. The other one is deterministic and monodisperse such that clusters of a given size $s_{0}$ are separated form neighboring clusters by a distance $l_{0}$ resulting in the concentration $\phi=s_{0} /\left(s_{0}+l_{0}\right)$.

After discussing the universality or nonuniversality of the persistences we concentrate on the dependence of the persistence exponents on the diffusion exponent $\gamma$ in section $\mathrm{VC}$. Thereafter we consider the size interval distributions (Sec. $\mathrm{VD}$ ). We demonstrate how the poor dynamic scaling of the interval distributions is a consequence of the two competing length scales $\mathcal{L}_{D}$ and $\mathcal{L}_{p}$. The scaling function of the region size distributions is shown to be of the simple exponential form in section $\mathrm{VE}$. 

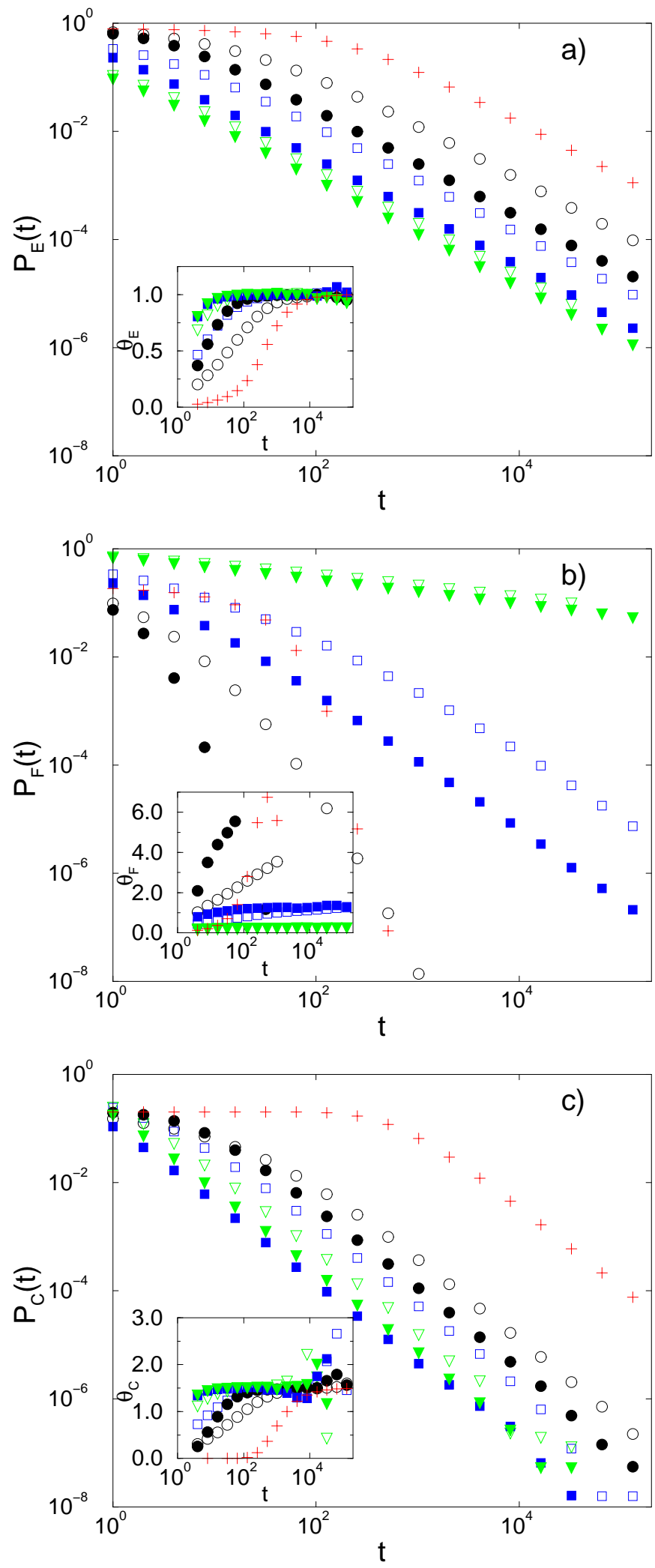

FIG. 5. The persistence probabilities a) $P_{E}(t)$, b) $P_{F}(t)$, and c) $P_{C}(t)$ for size independent diffusion coefficients $(\gamma=0)$ and for concentrations $\phi=0.20(\bigcirc), 0.50(\square)$, and $0.80(\nabla)$. Results from simulations using random and monodisperse $\left(s_{0}=1\right)$ initial conditions are denoted by open and filled symbols, respectively. Pluses $(+)$ are obtained with monodisperse initial conditions for $\phi=0.20$ and $s_{0}=10$. The insets show the running exponents.

\section{A. Concentration dependence}

We first analyze which persistence probabilities depend on concentration. We will present data only for size independent diffusion coefficients but we have checked that for $\gamma \neq 0$ the persistence probabilities behave in a similar way. Figure 5 shows the cluster, empty site, and filled site persistence probabilities for three different concentrations. A change in the concentration affects only the amplitudes of the cluster and empty site persistence distributions. The numerical estimates for the concentration independent persistence exponents obtained from the saturated part of the running exponents are $\theta_{C}(0)=1.48 \pm 0.03$ and $\theta_{E}(0)=1.00 \pm 0.02$. These both are in an excellent agreement with the exact results $\theta_{C}(0)=\frac{3}{2}$ and $\theta_{E}(0)=1$.
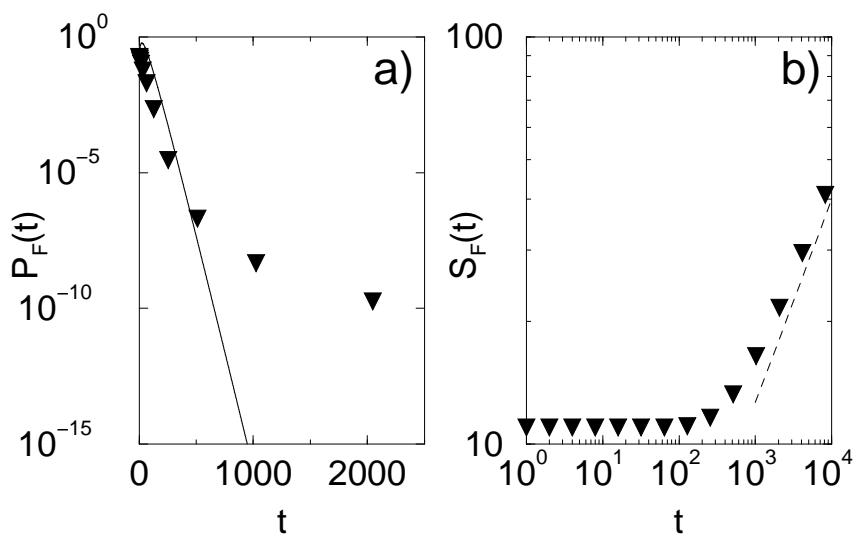

FIG. 6. a) Filled site persistence probability and b) average cluster size of those clusters which contain persistent sites for deterministic initial conditions with $\gamma=0, s_{0}=11$, and $\phi=0.2$. The solid line is given by equation (17) and the dashed line shows $t^{1 / 2}$ behavior.

For the filled site persistence the effect of a concentration change is much more pronounced. The distribution shows a transition from an algebraic decay to a relatively faster one when decreasing the concentration. This is in a qualitative agreement with the analysis of section III D but the agreement is not quantitative. In fact, the nonalgebraic decay seen in simulations is only a cross-over behavior and eventually the persistence probability will follow a power-law.

The discrepancy between the analysis of Sec. IIID and simulations can be traced to the assumption, that the 
clusters containing persistent filled sites have not aggregated. This is true only for times smaller than the average collision time between clusters, $t_{\text {coll }} \sim 1 /\left(D \phi^{2}\right)$, which indeed diverges for $\phi \rightarrow 0$. However, for any nonzero concentration there will be a time after which the collisions become important. In fact, the large time persistence probability of filled sites is dominated by the clusters, which have collided with other ones. This is illustrated in Figure 6, which shows both the persistence probability and the average size of those clusters which contain persistent sites. There is a clear crossover from the behavior given by the analysis of Sec. IIID at $t \approx 300$ to the one for which the collisions are significant. After this crossover time, the clusters including persistent empty sites grow similarly as the other ones. This is illustrated in figure $\mathrm{b}$ ), which shows that the average size of these clusters grows like $t^{1 / 2}$ at late times.

\section{B. Dependence on initial conditions}

Figure 5 also compares the persistence probabilities for the two initial conditions: random and monodisperse deterministic. A change in the initial condition does not have a significant effect on any of the persistence probabilities. It only affects the overall amplitude but leaves the qualitative behavior unaltered.

Simulations can of course not prove the universality of empty or cluster persistence but together with the arguments given in section III they strongly support this. However, they indisputably show that the filled site persistence is nonuniversal. Although non-universal, the empty site persistence is closely related to the persistence of bubbles in soap froths, which show similar concentration dependent behavior [27]. It would be interesting to study the similarities between these two persistences, but we will omit it here.

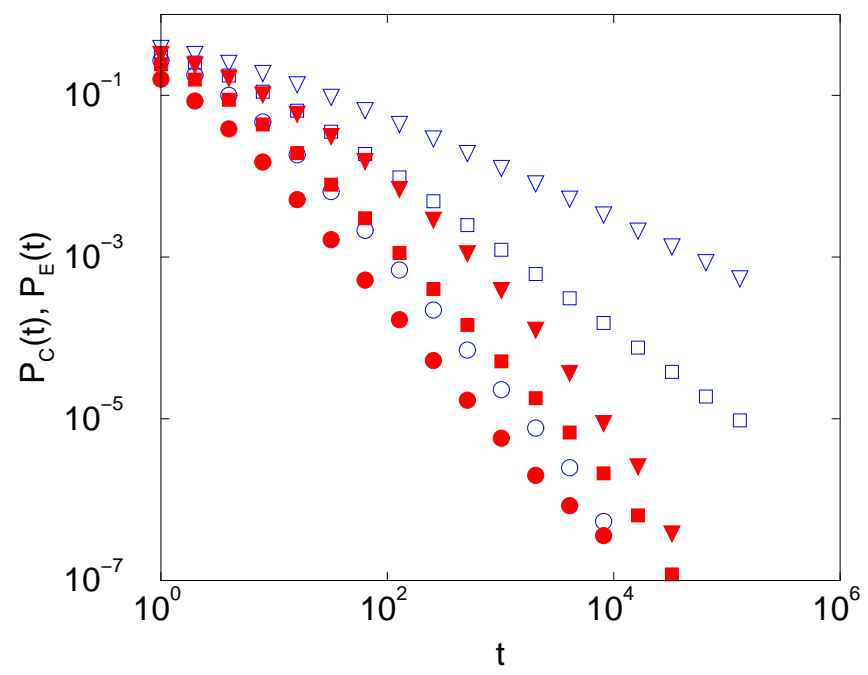

FIG. 7. The cluster and empty site persistence probabilities $P_{C}(t)$ (filled symbols) and $P_{E}(t)$ (empty symbols) for $\gamma=-1.0(\nabla), 0.0(\square)$, and $0.75(\bigcirc)$.

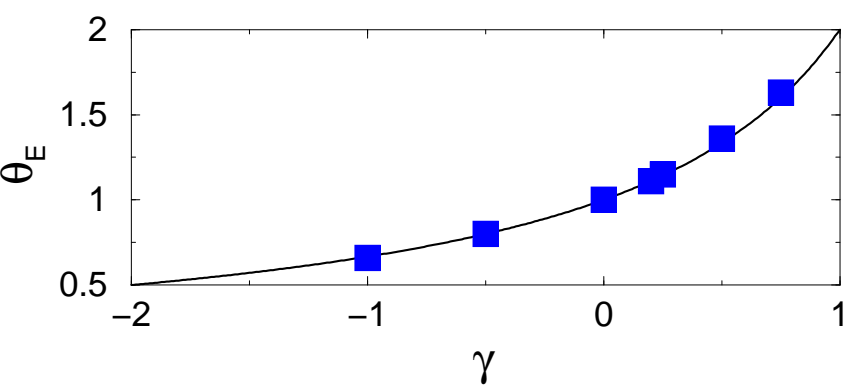

FIG. 8. Comparison of the numerically obtained empty site persistence exponent $\theta_{E}(\square)$ to the mean-field result $\theta_{E}=2 z=2 /(2-\gamma)$ (solid line) as a function of the diffusion exponent $\gamma$.

\section{Dependence on $\gamma$}

Thus far we have considered only systems with $\gamma=0$. All the dynamic scaling properties depend on $\gamma$ and the same is true for the persistence probabilities as figure 7 shows. The empty site persistence probability decays algebraically for all values of $\gamma<2$, above which the system gels in a finite time. The analytical prediction, $\theta_{E}=2 z$, obtained by replacing the discrete changes in the sizes of clusters besides the persistent one by a diffusion process, is compared to simulations in figure 8. The agreement is excellent, which basically means, that the system self-averages away the discreteness of the collision times. It also tells that there are no nontrivial correlations such that the clusters surrounding persistent empty sites would grow differently than the other ones.

The cluster persistence decays algebraically only for $\gamma \geq 0$ and faster than any power of $t$ for $\gamma<0$. The nonalgebraic behavior is not surprising on the basis of the discussion presented in section IIIB. Although a detailed study of the cluster persistence confirming the results presented in section [II B will be presented elsewhere [50], we briefly comment on the simulational problems one can encounter.

First, as the persistence exponent is related to the polydispersity exponent through $\theta_{C}=(2-\tau) z$, we are faced with same simulation problems than in the studies of $\tau$ (see e.g. 40,49]). Also the crossover time to the asymptotic behavior is large for $\gamma$ positive but close to zero. In order to see the real asymptotic behavior there must be very large clusters in the system. Consider, for example, $\gamma=0.2$, which still is not very small. The diffusion coefficient of a persistent cluster is of order $D_{1}$ and that of a typical cluster $D_{1} S(t)^{\gamma}$. The ratio of these two to be of order 10 , say, requires $S(t) \approx 10^{1 / \gamma} \approx 10^{5}$, which corresponds to a crossover time $t_{\text {cross }} \approx 10^{9}$. Even reaching this in simulations would be hard. This problem can, of course, be overcome by using a more sophisticated algorithm. We refer to 50 for more details on the cluster persistence. 


\section{Intervals between Consecutive Persistent Sites}

In section IV we argued that the size distribution of intervals between persistent quantities would scale according to $n_{X}(k ; t)=K_{X}(t)^{-2} f_{X}\left(k / K_{X}(t)\right)$ with a simple exponential scaling function [Eq. (28)]. When the corresponding persistence probability decays algebraically, $K_{X}(t) \sim A_{X} t^{\theta_{X}}$, with a nonuniversal amplitude $A_{X}(\phi)$, this can be presented also as $n_{X}(k ; t)=t^{-2 \theta_{X}} \tilde{f}_{X}\left(k / t^{\theta_{X}}\right)$.

The difference between these two scaling forms is that for the latter the scaling functions will not overlap each other for different concentrations due to the nonuniversal amplitude dependence. Therefore we show for clarity the scaling plots using the latter formulation. Furthermore, we prefer to show the scaling of the complement of the cumulative distribution

$$
I_{X}(k ; t)=\sum_{i \geq k} n_{X}(i ; t)
$$

which is much smoother due to the summation. It is easy to see from the scaling of $n_{X}(k ; t)$ and Eq. (28) that this distribution should scale as $I_{X}(k ; t)=t^{-\theta_{X}} g_{X}\left(k / t^{\theta_{X}}\right)$ with $g_{X}(x)=A_{X}^{-1} e^{-A_{X}^{-1} x}$.

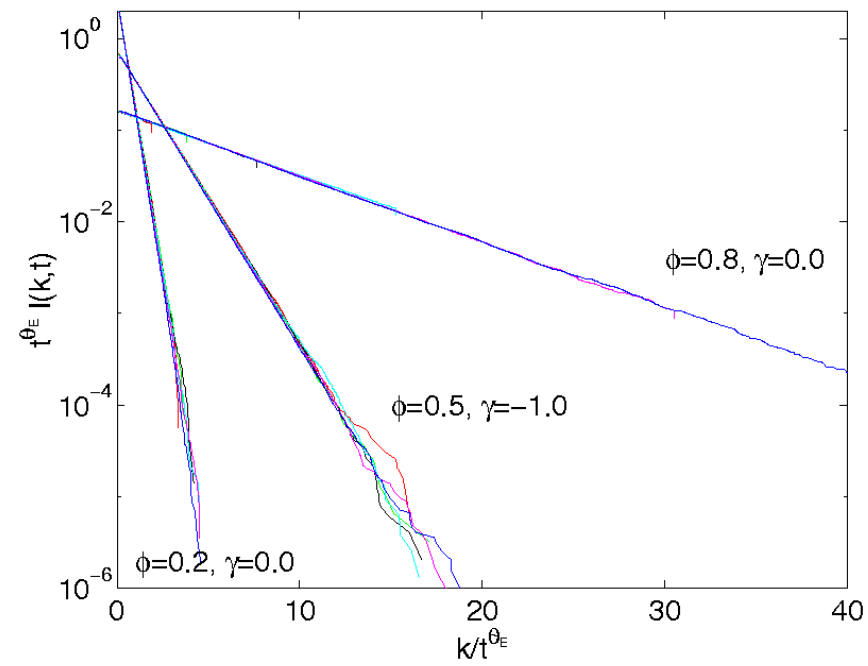

FIG. 9. Scaling plot for the complement of the cumulative distribution of intervals between two consecutive persistent empty sites at $t=2^{9}, \ldots, 2^{14}$ for concentrations and diffusion exponents shown in the figure. The value used for the persistence exponent $\theta_{E}=2 /(2-\gamma)$.

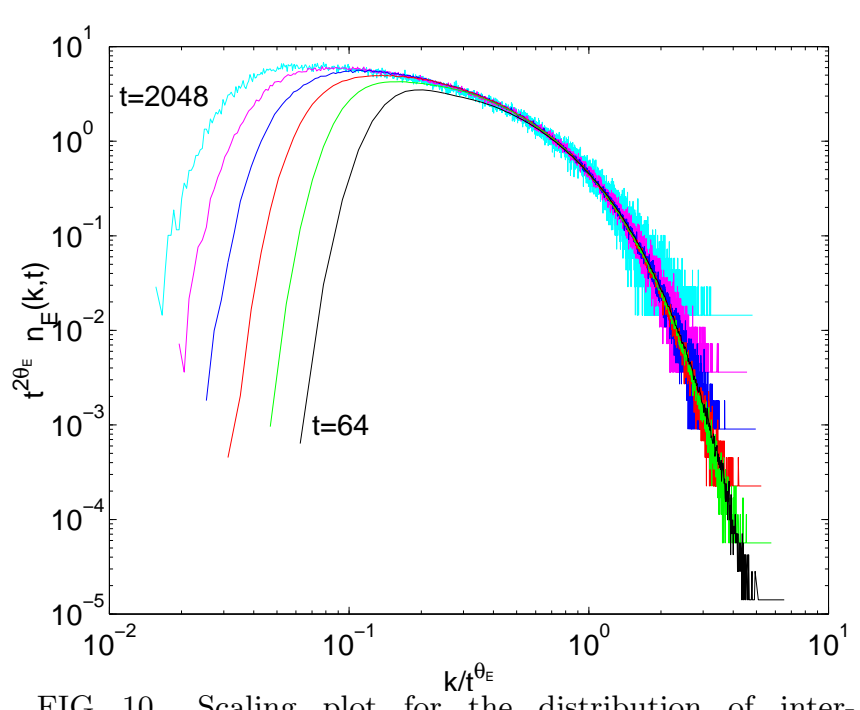

FIG. 10. Scaling plot for the distribution of intervals between two consecutive persistent empty sites at $t=2^{6}, \ldots, 2^{11}$ for $\phi=0.2$ and $\gamma=0$.

Figure 9 shows that the scaling works for the empty site persistence and that the scaling function is an exponential one indeed. The plots for the other persistence definitions are similar and are not shown. The scaling function is universal and all the curves in figure 9 would overlap each other if one would plot $K_{E}(t) I(k ; t)$ as a function of $k / K_{E}(t)$. Note, that the diffusion exponent $\gamma$ has no influence on the scaling function.

Although the summation in Eq. (30) smoothens the data it at the same time loses information about the small $k / t^{\theta_{E}}$ behavior. This is illustrated in figure 10 where no summation is done. For $k \ll t^{\theta_{E}}$ the scaling does not work. The reason is the following. The scaling should work in the limit $k \rightarrow \infty$ and $t \rightarrow \infty$ with $y=k / t^{\theta_{E}}$ fixed. Especially, the condition $k \gg t^{z}$ should be satisfied for the two length scales of the problem to be well separated. Define now a time-dependent $y_{c}(t)$ so that the scaling works for $y>y_{c}(t)$. This quantity gets smaller at the same rate as the curves in figure 10 shift towards zero. Our estimate from the numerical data gives $y_{c}(t) \sim t^{-0.50 \pm 0.03}$, which is consistent with $y_{c}(t) \sim t^{-z}$. Thus the poor scaling in figure 10 for $k \ll t^{\theta_{E}}$ is just a manifestation of the finite time behavior with two competing length scales, $\mathcal{L}_{D}$ and $\mathcal{L}_{p}$. This effect vanishes in the scaling limit. A similar, although not as clear, violation of scaling induced by the same reason is seen in the study of persistence in the $q$-state Potts model 断. 


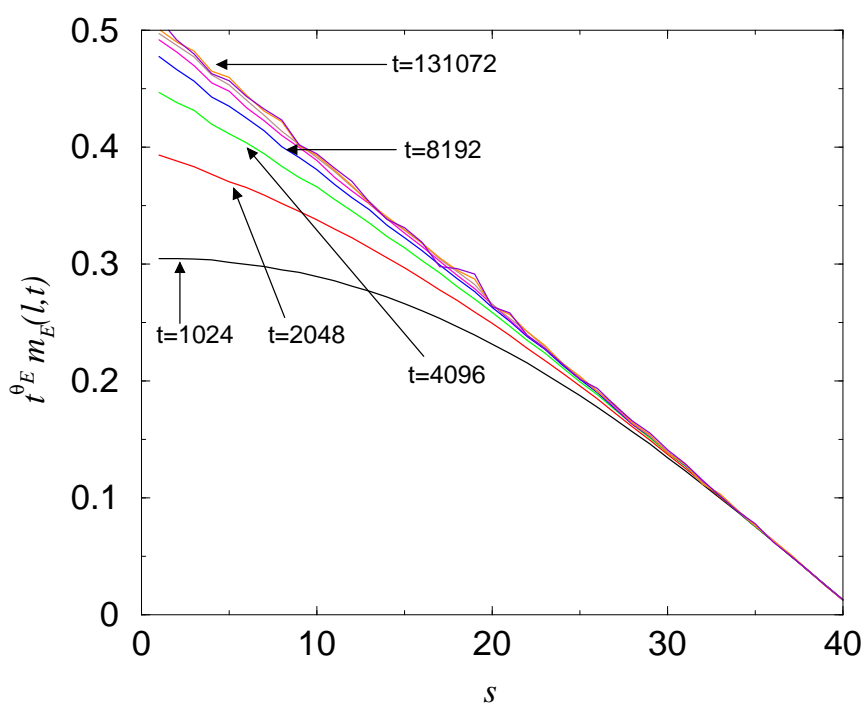

FIG. 11. The size distributions of persistent empty regions for $\gamma=0$ and ordered initial conditions with $s_{0}=10$ and $\phi=0.2$. The time instances range from $t=2^{10}$ to $t=2^{17}$.

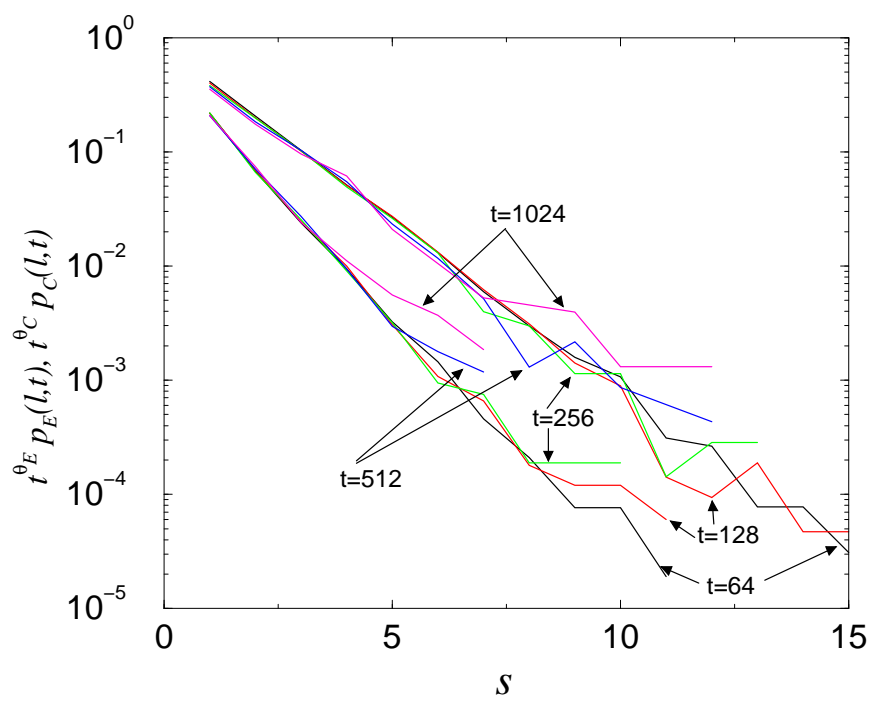

FIG. 12. The size distributions of persistent empty regions (upper curves) and clusters (lower curves) for random initial conditions and $\gamma=0.75$. The measurement times are shown in the figure.

\section{E. Persistent Regions}

The mean-field arguments used for the empty site exponent predicted the region size distribution to depend on the initial cluster size distribution. Calculations indicated a linear [see Eq. (10)] and an exponen-

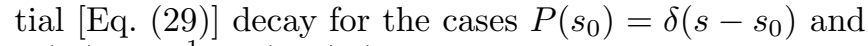
$P\left(s_{0}\right)=s_{0}^{-1} \exp \left(-s / s_{0}\right)$, respectively. It was further argued that the dependence on the diffusion exponent enters only through a multiplicative factor of $t^{-2 /(2-\gamma)}$ in the distributions. This in turn means that the average region size would approach a constant at late times. Figures 11 and 12 show the empty region distributions for these two cases and confirm the predictions.

In Figure 11 the smallest times shown are not large enough for the analysis of section IIIC to be valid but the tendency of the distribution to approach a straight line is clearly visible. The reason why the asymptotic behavior is reached earlier in Figure 12 is that the important measure is not the time itself. Rather it is the ratio of the diffusion coefficients of clusters, which naturally grows faster the larger the diffusion exponent $\gamma$ is. Note, that in neither of the figures the horizontal axes have been scaled, which simply means that the average region size is constant, i.e., independent of time. This is consistent with the arguments presented in the section IV. The region size distribution between persistent filled sites behaves in the same way with a concentration dependent exponent $\theta_{F}$.

As discussed at the end of section IV the size distribution of persistent clusters remains unaltered both for monodisperse initial condition and for $\gamma=0$. Therefore we only present the result for random initial conditions and for $\gamma=0.75$ in figure 12. The scaling is the same as it is for $\gamma=0$, i.e., the scaling function is a pure exponential and the average cluster size is asymptotically independent of time.

\section{CONCLUSIONS}

In this paper we have considered persistence in an aggregation process, in the simple case of one-dimensional DLCA. The emphasis is on local properties: cluster, empty site and filled site persistence together with the corresponding region and interval size distributions. We have shown that the three persistences are independent of each other with each behaving in its own, specific way.

The perhaps most natural choice, the probability that a site has remained in the same state (either filled or empty), turns out to be nonuniversal. Nevertheless, only one of the two contributing probabilities, the filled site persistence, is responsible for this fact. The other one, the empty site persistence, is universal. In the DCLA the difference in the dynamics of empty and filled sites implies that there is no symmetry that would produce similarity in the persistence behaviors. The reason why the cluster persistence is a third independent quantity derives from the fact that it is a cluster property whereas the two other persistence definitions are considered at a fixed point in space.

To summarize, the filled site persistence decays asymptotically algebraically for $\gamma<2$ and for all concentrations: $P_{F}(t) \sim t^{-\theta_{F}}$. The persistence exponent $\theta_{F}$ depends on concentration and is therefore nonuniversal. At low concentrations the filled site persistence decays as a stretched exponential upto a concentration dependent crossover time after which the collisions between clusters 
become important and start to dominate the persistence behavior.

The empty site persistence is universal. Although we have not been able to prove this, the universality is supported both by mean-field type continuum arguments and by computer simulations. The former leads to a relatively simple relation between the persistence exponent and the dynamic exponent, $\theta_{E}=2 z$, verified by simulations. This is one of the few examples [4], where the inequality $\theta>z d$, where $d$ is the spatial dimension, is fulfilled. The consequence of this is that the persistent empty regions do not have a fractal character. This is not true for example for the persistent regions in the Ising 10,4] or diffusion-annihilation [17] models. The fact that $\theta_{E}$ is notably larger than $z$, makes the separation of the diffusive and persistence length scales clearly visible in the DLCA.

The cluster persistence probability decays algebraically only for $0 \leq \gamma<2$ and as stretched exponentially for $\gamma<0$. For $0<\gamma<2$ the persistence exponent is given by $\theta_{C}(\gamma)=2 z=2 /(2-\gamma)$ and it is discontinuous as $\gamma \rightarrow 0^{+}$since $\theta_{C}(0)=\frac{3}{2}$. All these results are in close connection with the scaling of the cluster size distribution, especially with the small $x$ decay of the scaling function $f(x)$. In fact, there is a scaling relation between the exponents $\theta_{C}=(2-\tau) z$. This independence of the cluster persistence probability on concentration and the strong support of the universal dynamic scaling behavior of the cluster size distribution [42] speak for the universality of the cluster persistence. The scaling relation together with the result for $\theta_{C}$ offers a new way to approach the determination of $\tau$ for DLCA.

It is worth emphasizing that the universal empty and cluster persistences decay with a same exponent $\theta_{E}=$ $\theta_{C}=2 z=2 /(2-\gamma)$ for $0<\gamma<2$ but have nothing to do with each other for $\gamma<0$. In fact, for $\gamma \geq 0$ we can write $P_{C}(t) \sim\left[P_{E}(t)\right]^{\Gamma}$, where $\Gamma$ takes the values $3 / 2$ and 1 for $\gamma=0$ and $0<\gamma<2$, respectively. A similar relation, $P_{C}(t) \sim\left[P_{E}(t)\right]^{\Gamma}$, with a non-trivial $\Gamma$ has been observed also for noninteracting random walkers [19] and for the Potts model [12] in one dimension. In DLCA the same persistence exponents, i.e., $\Gamma=1$, for positive $\gamma$ are due to the fact that the clusters giving the dominant contribution to the cluster persistence are those which remain stationary. For other values of $\Gamma$ the interpretation is more opaque.

Although the persistences decay in a different manner (algebraic or faster) the intervals between persistent regions scale similarly for all cases: $n_{X}(k ; t)=$ $K_{X}(t)^{-2} f_{X}\left(t / K_{X}(t)\right)$. This is due to the relation $\theta_{X}>$ $z$, which causes the persistent regions to be well separated at late times. Therefore the regions survive independently and the intervals size distribution is a simple exponential $f_{X}(y)=e^{-y}$.

The size distributions of persistent regions depend on the initial cluster size distribution. They do not depend on the diffusion exponent which only enters through a multiplicative time dependence on the distribution, for example, $p_{E}(l, t) \sim t^{-2 /(2-\gamma)} \tilde{p}_{E}(l)$. This leads to a constant average region size at late times, which can be estimated from the knowledge of the initial cluster size distribution.

In conclusion, we have presented a rather comprehensive study of various local persistence probabilities in the one-dimensional DLCA. Our study is of interest also for the sake of practical realizations. Aggregation processes are plentiful, and all of the definitions - whether a point in space is occupied or not by a cluster, or whether clusters survive intact - might well be possible to measure in experimentally. It is interesting also to note that when the decay of a persistence probability turns out to be algebraic and universal, the exponent is always directly in some relation to the dynamical exponent $z$ of the aggregation process. It is an obvious question to ask how the various quantities work out in higher dimensions. This generalization seems both challenging and interesting.

\section{ACKNOWLEDGMENTS}

The authors thank P. E. Salmi for numerous discussions. E. K. O. H. also thanks S. Majaniemi for helpful remarks. This research has been supported by the Academy of Finland's Center of Excellence program.

[1] S. N. Majumdar, Curr. Sci. (India) 77, 370 (1999).

[2] S. N. Majumdar, C. Sire, A. J. Bray, and S. J. Cornell, Phys. Rev. Lett. 77, 2867 (1996).

[3] B. Derrida, V. Hakim, and R. Zeitak, Phys. Rev. Lett. 77, 2871 (1996).

[4] A. J. Bray and S. J. O'Donoghue, Phys. Rev. E 62, 3366 (2000).

[5] B. Derrida and R. Zeitak, Phys. Rev. E 54, 2513 (1996).

[6] P. L. Krapivsky and E. Ben-Naim, Phys. Rev. E 56, 3788 (1997).

[7] E. Ben-Naim and P. L. Krapivsky, J. Stat. Phys. 93, 583 (1998).

[8] C. Sire and S. N. Majumdar, Phys. Rev. E 52, 244 (1995).

[9] V. Spirin, P. L. Krapivsky, and S. Redner, Phys. Rev. E 60, 2670 (1999).

[10] S. Jain and H. Flynn, J. Phys. A 33, 8383 (2000).

[11] D. S. Fisher, P. L. Doussal, and C. Monthus, Phys. Rev. Lett. 80, 3539 (1998).

[12] S. J. O'Donoghue and A. J. Bray, preprint, condmat/0111159.

[13] S. J. O'Donoghue and A. J. Bray, Phys. Rev. E 64, 041105 (2001).

[14] G. Manoj and P. Ray, preprint, cond-mat/9901130.

[15] E. Ben-Naim and P. L. Krapivsky, preprint, condmat/9902073.

[16] G. Manoj and P. Ray, preprint, cond-mat/9902339.

[17] G. Manoj and P. Ray, J. Phys. A 33, L109 (2000). 
[18] G. Manoj and P. Ray, J. Phys. A 33, 5489 (2000).

[19] S. J. O'Donoghue and A. J. Bray, preprint, condmat/0111133.

[20] E. Ben-Naim, L. Frachebourg, and P. L. Krapivsky, Phys. Rev. E 53, 3078 (1996).

[21] M. Howard and C. Godrèche, J. Phys. A 31, L209 .

[22] S. N. Majumdar and A. J. Bray, Phys. Rev. Lett. 86, 3700 (2001).

[23] H. Kallabis and J. Krug, Europhys. Lett. 45, 20 (1999).

[24] J. Krug et al., Phys. Rev. E 56, 2702 (1997).

[25] Z. Toroczkai, T. J. Newman, and S. D. Sarma, Phys. Rev. E 60, R1115 (1999).

[26] W. Y. Tam, R. Zeitak, K. Y. Szeto, and J. Stavans, Phys. Rev. Lett. 78, 1588 (1997).

[27] W. Y. Tam, A. D. Rutenberg, B. P. Vollmayr-Lee, and K. Y. Szeto, Europhys. Lett. 51, 223 (2000).

[28] M. Marcos-Martin et al., Physica A 214, 396 (1995).

[29] G. P. Wong, R. W. Mair, and R. L. Walsworth, Phys. Rev. Lett. 86, 4156 (2001).

[30] B. Yurke, A. N. Pargellis, S. N. Majumdar, and C. Sire, Phys. Rev. E 56, R40 (1997).

[31] B. Derrida, V. Hakim, and V. Pasquier, Phys. Rev. Lett. 75, 751 (1995).

[32] B. Derrida, V. Hakim, and V. Pasquier, J. Stat. Phys. 85, 763 (1996).

[33] T. W. Burkhardt, J. Phys. A 26, L1157 (1993).

[34] A. Baldassarri, J. P. Bouchaud, I. Dornic, and C. Godrèche, Phys. Rev. E 59, R20 (1999).

[35] P. Meakin, Phys. Scripta 46, 295 (1992).

[36] J. L. Spouge, Phys. Rev. Lett. 60, 871 (1988).

[37] E. V. Albano and M. A. Munoz, Phys. Rev. E 63, 031104 (2001).
[38] O. Deloubriere, J. Phys. A 33, 7025 (2000).

[39] P. G. J. van Dongen and M. H. Ernst, Phys. Rev. Lett. 54, 1396 (1985).

[40] K. Kang, S. Redner, P. Meakin, and F. Leyvraz, Phys. Rev. A 33, 1171 (1986).

[41] S. Miyazima, P. Meakin, and F. Family, Phys. Rev. A 36, 1421 (1987).

[42] E. K. O. Hellén, T. P. Simula, and M. J. Alava, Phys. Rev. E 62, 4752 (2000).

[43] D. ben-Avraham, in Nonequilibrium Statistical Mechanics in One Dimension, 1st ed., edited by V. Privman (Cambridge University Press, Cambridge, 1997), pp. 2950.

[44] S. N. Majumdar and S. J. Cornell, Phys. Rev. E 57, 3757 (1998).

[45] M. E. Fisher, J. Stat. Phys. 34, 667 (1984).

[46] M. E. Fisher and M. P. Gelfand, J. Stat. Phys. 53, 175 (1988).

[47] P. L. Krapivsky and S. Redner, J. Phys. A 29, 5347 (1996).

[48] P. G. J. van Dongen and M. H. Ernst, J. Phys. A 18, 2779 (1985).

[49] S. Cueille and C. Sire, Phys. Rev. E 55, 5465 (1997).

[50] E. K. O. Hellén, P. E. Salmi, and M. J. Alava, in preparation .

[51] S. Redner and P. L. Krapivsky, Am. J. Phys 67, 1277 (1999).

[52] G. H. Weiss, Aspects and Applications of the Random Walk, 1st ed. (North-Holland, Amsterdam, 1994), p. 201.

[53] L. P. Kadanoff, Statistical Physics: Statics, Dynamics and Renormalization, 1st ed. (World Scientific, Singapore, 2000), p. 360. 